\title{
Distribución del género Tityus Koch, 1836 (Scorpiones: Buthidae) en la ciudad de La Plata (Argentina)
}

\author{
Distribution of the genus Tityus Koch, 1836 (Scorpiones: Buthidae) in the city of La Plata \\ (Argentina)
}

Luis A. Giambelluca* 1

https://orcid.org/0000-0001-6530-8143

giambelluca@cepave.edu.ar

\section{Sandra E. González ${ }^{1}$}

https://orcid.org/0000-0002-3477-3453

sandra@cepave.edu.ar

Sergio G. Rodríguez Gil ${ }^{1}$

https://orcid.org/0000-0003-4608-1961 sergiorodriguezgil@cepave.edu.ar

\section{Alda González ${ }^{1}$}

https://orcid.org/0000-0002-2089-0907 asgonzalez@cepave.edu.ar

\section{*Corresponding author}

1 Centro de Estudios Parasitológicos y de Vectores CEPAVE (CONICET-UNLP Asoc. CICBA), Boulevard $120 \mathrm{~s} / \mathrm{n}$ entre Av. 60 y Calle 64 C.P. 1900, La Plata, Argentina.

\section{Citación}

Giambelluca LA., González SE, Rodríguez Gil SG, González A. 2021. Distribución del género Tityus Koch, 1836 (Scorpiones: Buthidae) en la ciudad de La Plata (Argentina). Revista peruana de biología 28(4): e21154 (Noviembre 2021). doi: http://dx.doi.org/10.15381/ rpb.v28i4.21154

$\begin{array}{ll}\text { Presentado: } & 03 / 09 / 2021 \\ \text { Aceptado: } & 02 / 11 / 2021 \\ \text { Publicado online: } & 26 / 11 / 2021 \\ \text { Editor: } & \text { Diana Silva }\end{array}$

\section{Resumen}

En la ciudad de La Plata se encuentra el género Tityus con registros desde 1943. Se estudió la distribución territorial del género Tityus en esta ciudad y su expansión en los últimos 15 años a partir de las consultas recibidas en el Laboratorio de Aracnología del CEPAVE, provenientes de particulares e instituciones públicas y privadas. Se recibieron 189 consultas desde el año 2005 al 2020. Se utilizó el programa QGIS para geolocalizar cada escorpión con capas de trazado urbano y desagües pluviales. Para el análisis espacial de distribución se consideró un home range de una hectárea por individuo para el cálculo de área y en los casos de áreas de influencias solapadas durante el mismo año se consideró el área como la unión de las mismas. El género Tityus en la ciudad de La Plata está representado por las especies T. carrilloi y $T$. confluens ambas de interés sanitario y de hábitos sinantrópicos, que ocupan dos zonas diferentes bien definidas. El análisis de la dispersión independiente en las dos zonas indicaría que podrían usar los desagües para dispersarse, y al ser inconexos estos no habría flujo de escorpiones entre ambas zonas. La colonización de estas especies en la zona en estudio se vio afianzada para $T$. confluens a partir del año 2005 y para T. carrilloi a partir del 2011. Los mayores registros son en los meses cálidos, desde enero a abril, siendo este último el de mayor valor. Septiembre es el único mes sin denuncia. La tasa de expansión calculada fue de 4.42 ha/año.

\section{Abstract}

In the city of La Plata occurs the genus Tityus with records dating back to 1943 . The territorial distribution of the genus Tityus in this city and its expansion was studied based on inquiries received at the CEPAVE Arachnology Laboratory from individuals and public and private institutions. 189 inquiries were received from 2005 to 2020. The QGIS program was used to geolocate each scorpion with urban layout layers and storm drains. For the spatial analysis of distribution, a home range of one hectare per individual was considered to calculate the area and in the cases of areas of overlapping influences during the same year, the area was considered as their union. The genus Tityus in the city of La Plata is represented by the species T. carrilloi and $T$. confluens, both of health interest and of synanthropic habits, which occupy two different well-defined areas. The analysis of the independent dispersion in the two zones would indicate that they could use the drains to disperse, and since they are unconnected there would be no flow of scorpions between both zones. The colonization of these species in the study area was strengthened for $T$. confluens as of 2005 and for T. carrilloi as of 2011. The highest records are in the warm months, from January to April, the latter being of higher value. September is the only month without complaints. The expansion rate calculated was $4.42 \mathrm{ha} / \mathrm{year}$.

Palabras claves:

Arácnidos urbanos; Tityus confluens; Tityus carrilloi; Escorpiones sinantrópicos; Escorpionismo.

Keywords:

Urban arachnids; Tityus confluens; Tityus carrilloi; Synanthropic scorpions; Scorpionism. 


\section{Introducción}

La ciudad de La Plata $\left(34^{\circ} 55^{\prime}\right.$ S: $57^{\circ} 57^{\prime} \mathrm{W}$ ), en la provincia de Buenos Aires, Argentina está enclavada en una división territorial del mismo nombre, en la cual coexisten dos familias de escorpiones: Bothriuridae y Buthidae. La primera, representada por Bothriurus bonariensis Koch, 1843, habita en zonas periurbanas y rurales y su veneno es inofensivo para el ser humano (de Roodt et al. 2019). Esta especie tiene una amplia distribución en Argentina y se diferencia fácilmente de las especies del género Tityus Koch, 1836 por tener pinzas cortas y abultadas y un telson simple (Ojanguren Affilastro, 2005). La segunda familia está representada por el género Tityus que es fácilmente identificable por tener pinzas largas y delgadas y una apófisis subaculear en el telson. Su distribución es americana, desde México hasta Argentina, siendo sus registros más australes los de la provincia de Buenos Aires (de Roodt et al. 2019). Para la ciudad de La Plata, Tityus está representado por dos especies de escorpiones de mediano tamaño, aproximadamente $7 \mathrm{~cm}$ : Tityus carrilloi Ojanguren-Affilastro, 2021 de coloración clara con tres bandas dorsales oscuras longitudinales y Tityus confluens Borelli, 1899 con coloración dorsal oscura uniforme. Tityus carrilloi fue descripta como nueva especie por Ojanguren-Affilastro et al. (2021) quienes señalan que Tityus trivittatus Kraepelin, 1898 no está presente en Argentina, siendo sus límites los ríos Paraná y Paraguay en territorio paraguayo y brasilero. Ambas especies presentes en La Plata son de hábitos sinantrópicos y de importancia sanitaria por sus venenos de acción neurotóxica que afecta al ser humano (de Roodt 2014, de Roodt et al. 2003, Ojanguren-Affilastro et al. 2021).

En el 2005 fueron incorporados dos ejemplares del género Tityus al Centro de Estudios Parasitológicos y de Vectores (CEPAVE, dependiente del Consejo Nacional de Investigaciones Científicas y Técnicas (CONICET) Universidad Nacional de La Plata (UNLP), asociado a la Comisión de Investigaciones Científicas de la Provincia de Buenos Aires (CICPBA)) el cual es un Centro de referencia para la determinación de arácnidos de la ciudad de La Plata. Desde esa fecha hasta la actualidad se ha observado un aumento sostenido de registros. La aparición e incremento de registro de escorpiones del género Tityus en la ciudad de La Plata ocurre luego de la inundación de la ciudad en el 2013. Es así que, desde el 2005 hasta la actualidad, en el Laboratorio de Aracnología del CEPAVE han sido efectuadas más de 200 consultas referidas a la presencia de escorpiones, lo que ha causado preocupación de las autoridades llegando a ser un tema tratado en la Cámara de Diputados de la provincia de Buenos Aires en su 9na sesión ordinaria del periodo 142 realizado el 13 de agosto de 2014 (Diputados 2014).

El objetivo de este trabajo es dar a conocer la distribución territorial del género Tityus en la ciudad de La Plata y su expansión a partir de las consultas recibidas en el CEPAVE provenientes de diversas instituciones y del público en general.

\section{Materiales y métodos}

Se analizaron los escorpiones del género Tityus que ingresaron al Laboratorio de Aracnología del CEPAVE a través de consultas de particulares, entidades públicas y privadas desde el 2005 hasta el 2020. El Laboratorio de Aracnología inicio el servicio de consultas en el año 2005. Los ejemplares fueron conservados individualmente en frascos con alcohol $96^{\circ}$ como parte de la colección. Con la información de las consultas se confeccionó una ficha con la ubicación, colector, fecha del hallazgo y sexo. Los datos fueron cargados en una hoja de cálculo con extensión dbf. Se utilizó el programa Qgis (versión 3.1) con el fin de geolocalizar cada escorpión con sus metadatos registrados. Para el análisis espacial de distribución se usaron capas de trazado urbano y desagües pluviales, estos últimos están por debajo de las zonas de distribución de los escorpiones y podrían ser uno de los factores que explicarían la distribución de ambas especies.

Para el cálculo del área de los ejemplares se consideró un home range de una hectárea por individuo, es decir un escorpión puede desplazarse un máximo de $100 \mathrm{~m}$, por considerar que se ajusta a lo observado en nuestra área de estudio y en concordancia con otros estudios de desplazamiento de escorpiones (Bibbs et al. 2014). En los casos de los escorpiones que tuvieron áreas de influencias solapadas durante el mismo año se consideró el área como la unión de las mismas.

Se realizaron dos tipos de análisis de los datos: 1 ) área total ocupada y delimitada por los registros en cada año; 2) la suma acumulativa del área del año actual más la de los años anteriores.

El material fue identificado con las claves taxonómicas de Ojanguren-Affilastro (2005) y con el trabajo de Ojanguren-Affilastro et al. (2021).

\section{Resultados}

Se recibieron 189 consultas entre el 2005 y 2020. Se observó que las denuncias de aparición fueron aumentando, de sólo dos ejemplares en el 2005 a 25 en el 2020, presentando en 2013 el mayor número de ejemplares (33), que se correspondió con una gran inundación en la ciudad (Fig. 1).

Los ejemplares del género Tityus revisados en las consultas correspondieron a dos especies: $T$. confluens $(\mathrm{n}=154)$ y T. carrilloi $(\mathrm{n}=32)$. Estas especies están establecidas en dos zonas bien definidas dentro de la ciudad. Tityus carrilloi está presente en el barrio de Los Hornos, periférico a la estación de trenes Gambier, conformado por construcciones de poca antigüedad y con sectores abandonados correspondientes a talleres de una línea de ferrocarril (Zona I); mientras que T. confluens se ubica en un área del centro antiguo de la ciudad (Zona II) (Fig. 2). Estas especies también fueron registradas ocasionalmente en otras zonas satélites y en poco número de ejemplares $(1-3)$ (Tabla 1).

Las primeras consultas registradas en el CEPAVE son de 2005 y corresponden a T. confluens, en tanto que las consultas de T. carrilloi comenzaron en 2011 (Tabla 1). 


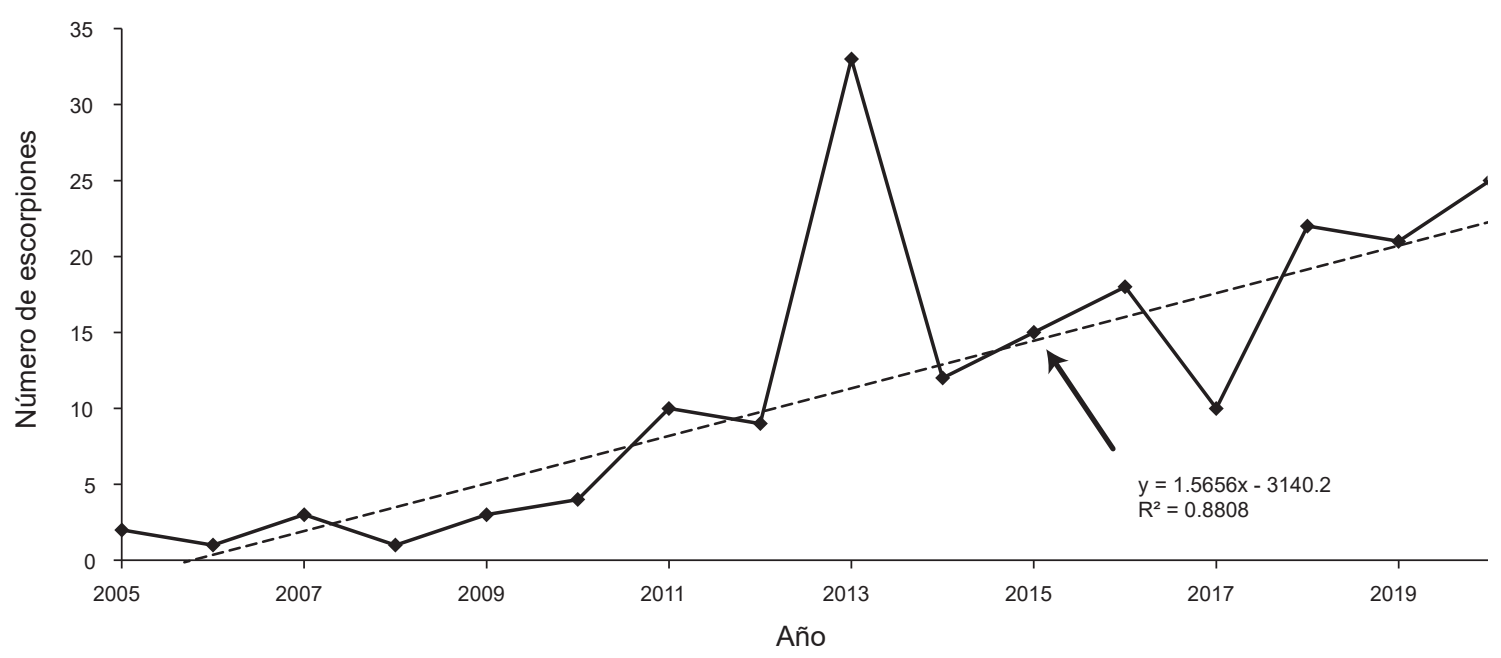

Figura 1. Número de registros del género Tityus por año en la ciudad de La Plata, Argentina. Datos basados en las consultas realizadas al CEPAVE desde 2005 a 2020. Línea punteada, tendencia de los datos, no incluye los valores del año 2013.

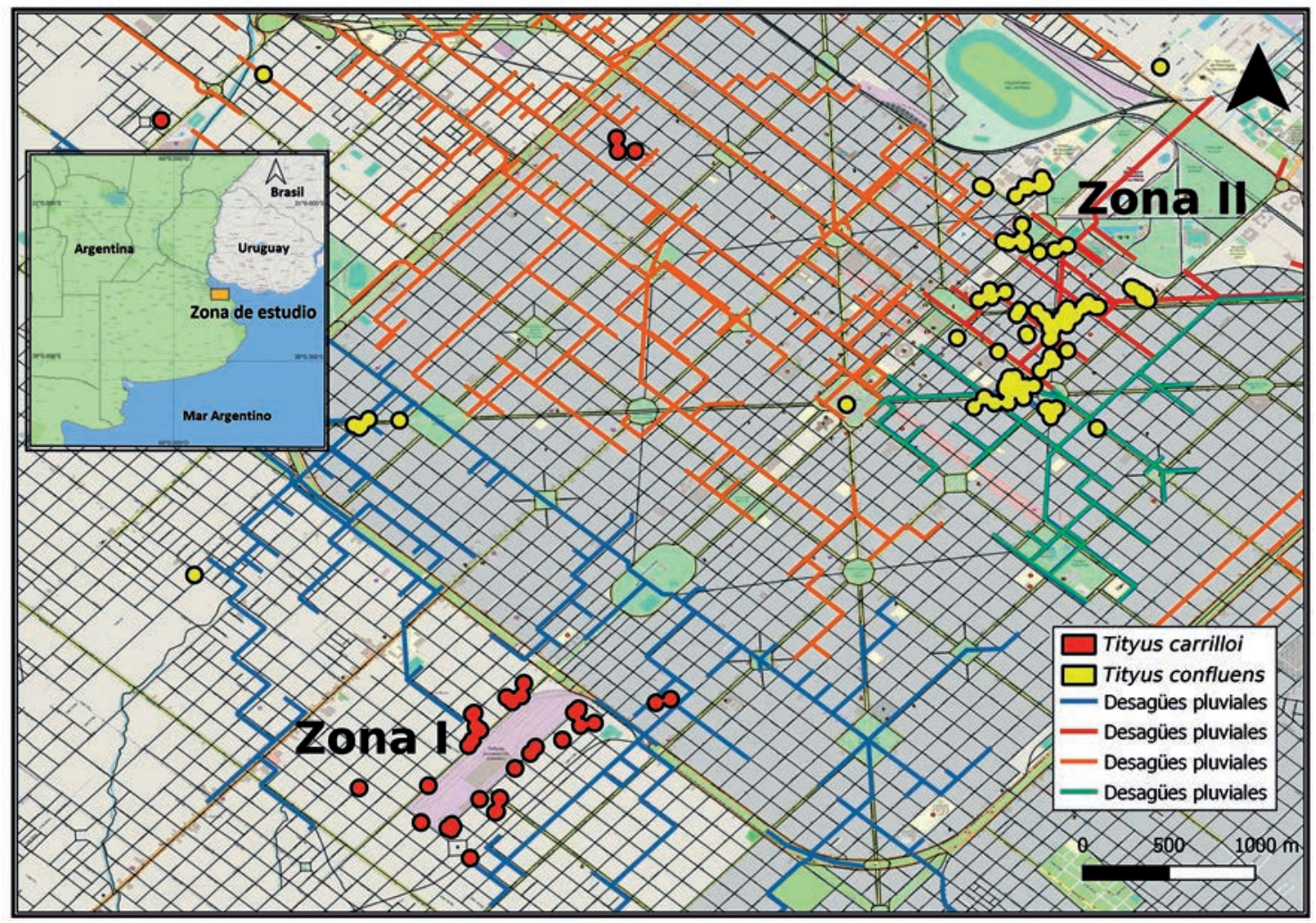

Figura 2: Áreas ocupadas por el género Tityus en la ciudad de La Plata, Argentina de acuerdo con las consultas realizadas en el CEPAVE desde 2005 a 2020. Zona I: Tityus carrilloi Barrio Los Hornos, Zona II: Tityus confluens Centro antiguo de la ciudad. Los distintos colores de las redes pluviales muestran diferentes ramales inconexos, correspondiendo el colora azul a la Zona I y los colores verde y rojo a la Zona II.

Tabla 1: Cantidad de reportes de individuos del género Tityus en el CEPAVE por año, desde 2005 a 2020, en zonas de la ciudad de La Plata, Argentina.

\begin{tabular}{|c|c|c|c|c|c|c|c|c|c|c|c|c|c|c|c|c|c|}
\hline Lugares & Especie & 2005 & 2006 & 2007 & 2008 & 2009 & 2010 & 2011 & 2012 & 2013 & 2014 & 2015 & 2016 & 2017 & 2018 & 2019 & 2020 \\
\hline \multirow{2}{*}{ Centro } & T. carrilloi & 0 & 0 & 0 & 0 & 0 & 0 & 0 & 0 & 0 & 0 & 0 & 0 & 0 & 0 & 0 & 0 \\
\hline & T. confluens & 2 & 1 & 1 & 1 & 3 & 4 & 8 & 7 & 30 & 11 & 9 & 12 & 7 & 15 & 18 & 13 \\
\hline \multirow{2}{*}{ Los Hornos } & T. carrilloi & 0 & 0 & 0 & 0 & 0 & 0 & 1 & 3 & 2 & 1 & 5 & 4 & 2 & 4 & 3 & 11 \\
\hline & T. confluens & 0 & 0 & 0 & 0 & 0 & 0 & 0 & 0 & 0 & 0 & 0 & 0 & 0 & 0 & 0 & 0 \\
\hline \multirow{2}{*}{ Otros } & T. carrilloi & 0 & 0 & 0 & 0 & 0 & 0 & 1 & 0 & 0 & 0 & 0 & 0 & 0 & 3 & 0 & 1 \\
\hline & T. confluens & 0 & 0 & 2 & 0 & 0 & 0 & 0 & 0 & 0 & 0 & 1 & 2 & 1 & 0 & 0 & 0 \\
\hline
\end{tabular}




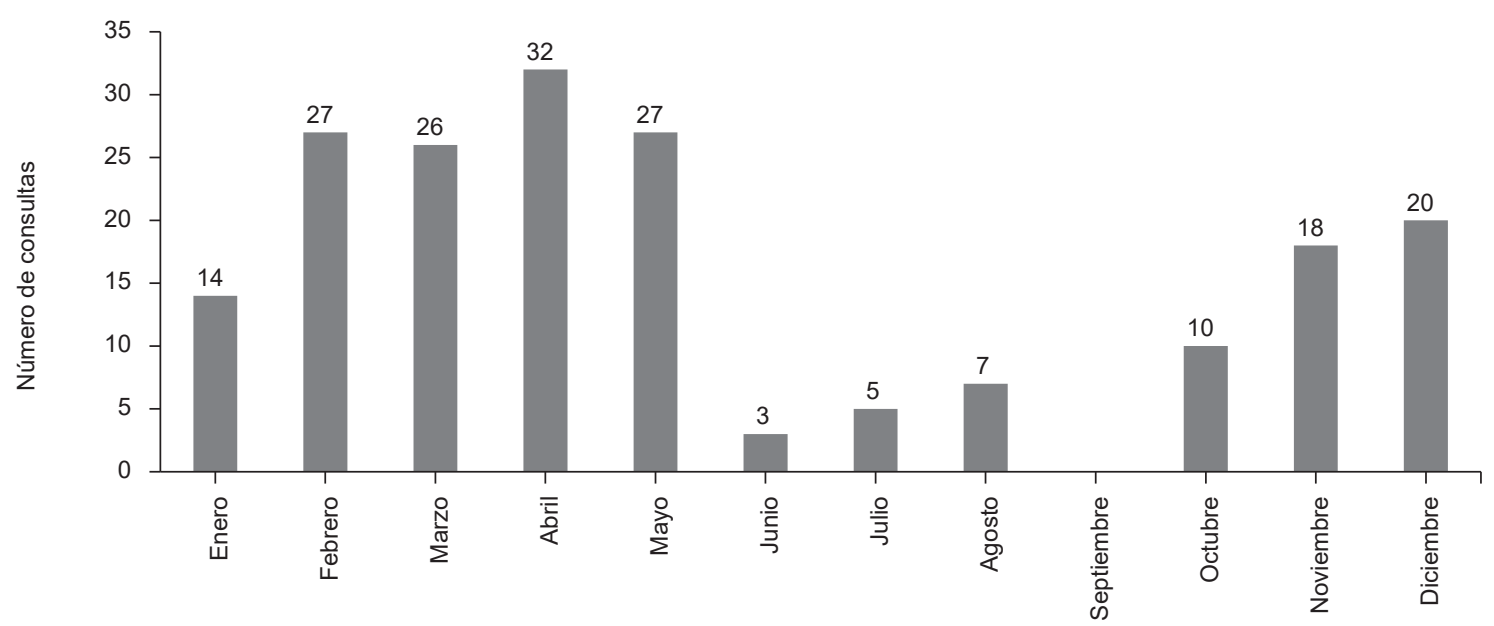

Figura 3: Consultas registradas en el CEPAVE desde 2005 a 2020, discriminadas por mes en la ciudad de La Plata, Argentina.

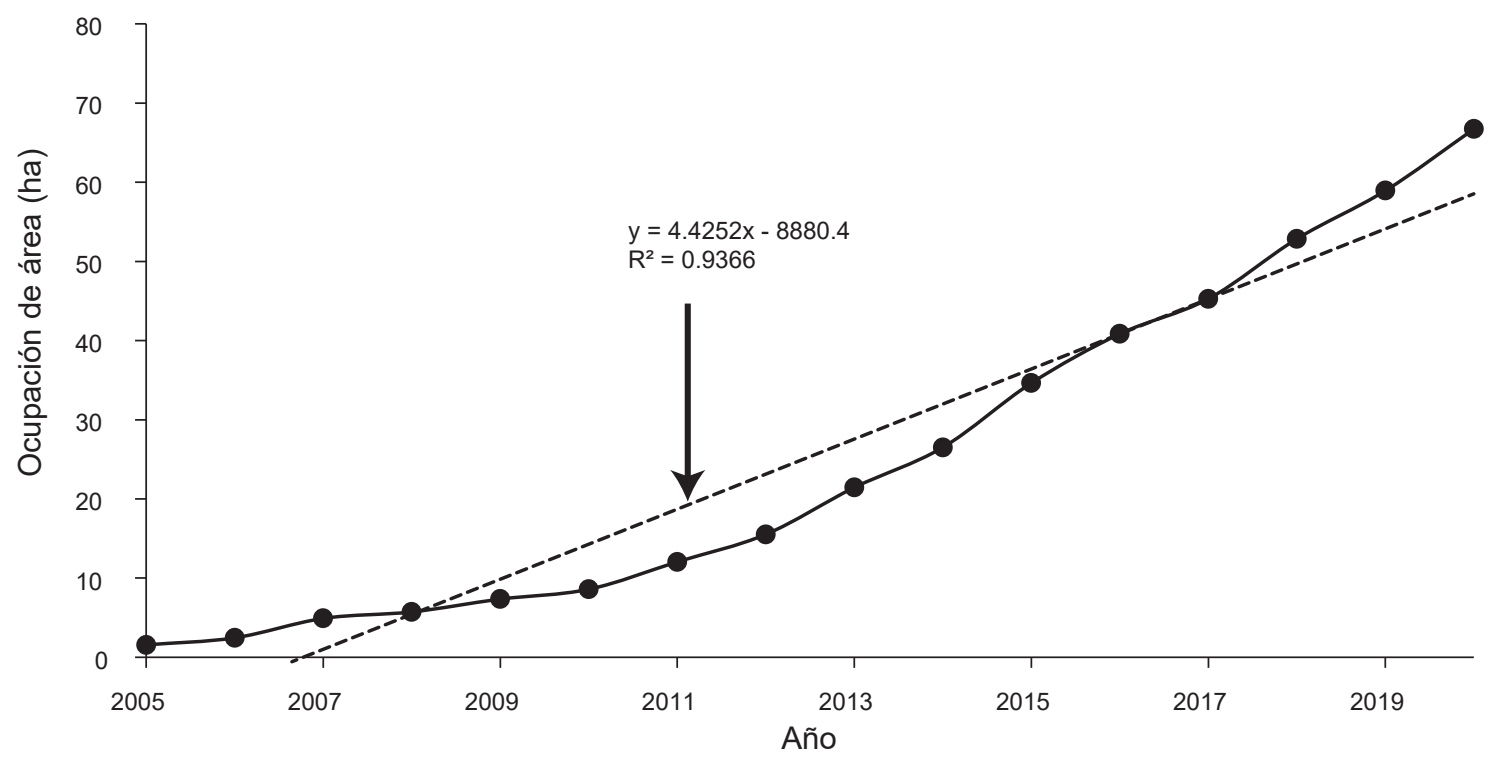

Figura 4: Área en hectáreas ocupadas por el género Tityus en la ciudad de La Plata, Argentina. Valores para áreas acumuladas de acuerdo con las consultas realizadas en el CEPAVE desde 2005 a 2020. Línea punteada, tendencia de los datos.

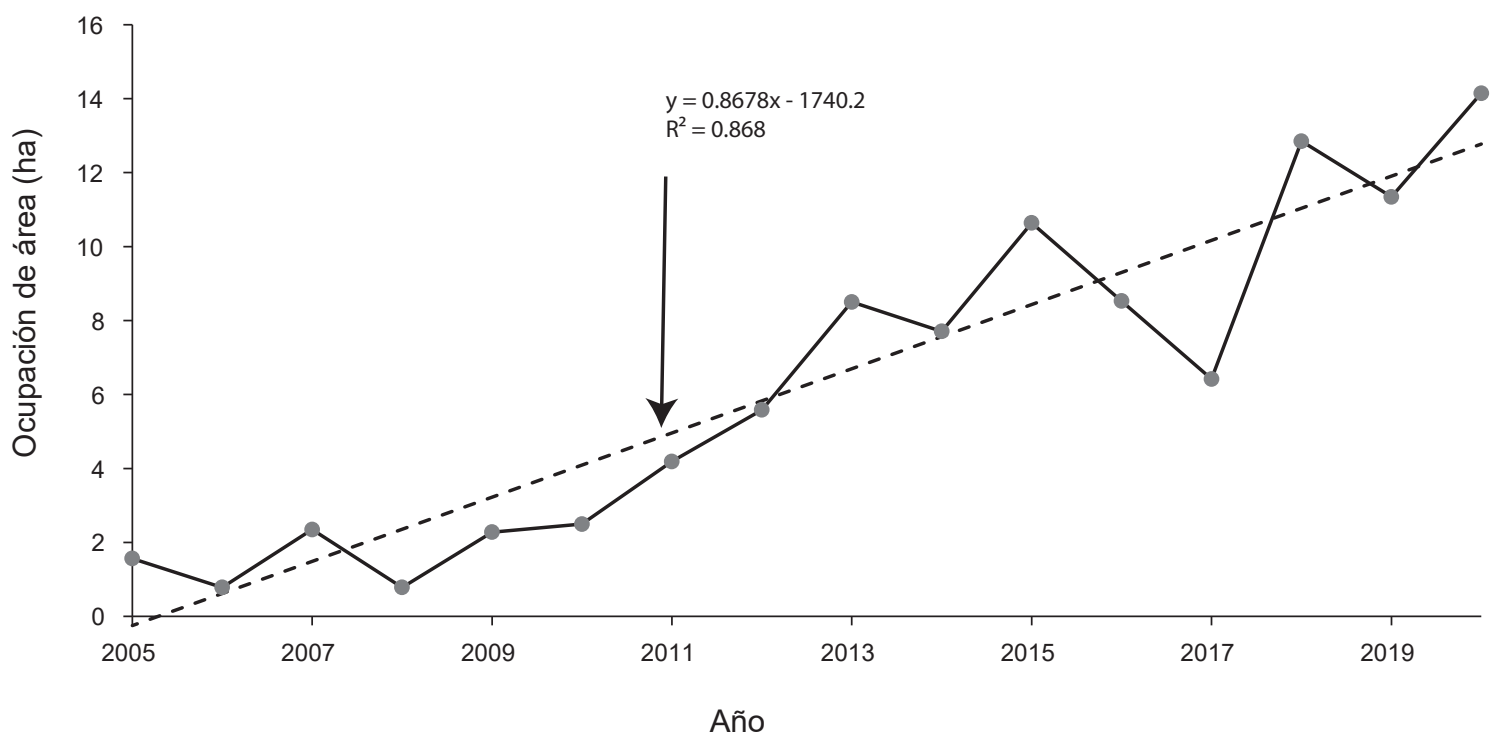

Figura 5: Área en hectáreas registrada en cada año por el género Tityus en la ciudad de La Plata, Argentina de acuerdo con las consultas realizadas en el CEPAVE desde 2005 a 2020. Línea punteada, tendencia de los datos. 
Todos los ejemplares de Tityus registrados durante los 15 años de estudio correspondieron a ejemplares hembras.

La presencia de individuos discriminada por meses a lo largo del año muestra mayor abundancia en los meses cálidos, desde enero a abril, siendo este último el de mayor registro. Septiembre es el único mes sin registros (Fig. 3).

Los valores de ocupación de área del género Tityus para la ciudad de La Plata, muestran un claro incremento al observarse el acumulado del área del año actual más la de los años anteriores y que se describe en la ecuación de la recta de $\mathrm{x}=4.4252 \mathrm{x}-8880.4$ (Fig. 4). También se observa el mismo patrón al analizar el área ocupada en cada año (Fig. 5).

\section{Discusión}

El género Tityus, en la ciudad de La Plata es urbano, su presencia se denuncia mayoritariamente en los meses cálidos y no tiene registros en septiembre. Esto coincide con lo señalado por Maury (1997) y con las observaciones registradas de i-Naturalist (Naturalista) para T. carrilloi, las cuales también muestran una disminución de hallazgos en los meses fríos.

El género Tityus fue citado por primera vez para La Plata por Roewer (1943). Pero, Moyano (2008) refiere una publicación en un diario de fecha 12 de enero de 1889 , sobre un accidente ocurrido en las calles 23 y 47 de La Plata donde falleció una persona por la picadura de un escorpión, y sugiere que podría haber sido del género Tityus. Según la bibliografía, desde el primer registro de Roewer pocos ejemplares de escorpiones han sido ingresados a colecciones con procedencia de La Plata. Los registros en colecciones corresponden a: un ejemplar depositado en el Senckenberg Museum Frankfurt (Alemania) SMF 9908047, colectado y citado por Roewer (1943); un ejemplar depositado en el Museo de La Plata (Argentina) MLP-ar 17366, colectado por Ringuelet en 1953 y citado por Maury (1970); un ejemplar depositado en el Museo Argentino de Ciencias Naturales (Argentina) MACN-Ar 6186, colectado por Garone en 1965 y citado por Maury (1970); un ejemplar depositado en el MACN-Ar 6190, colectado por Guinet en 1965 y citado por Maury (1970); un ejemplar depositado en el MACNAr sin número de colección, colectado por Giambelluca en 1990 y citado por Maury (1997). Luego se observa un período de ausencia de ingresos a colecciones con procedencia de la ciudad de La Plata hasta el 2005. Tomando en cuenta los registros en colecciones, las apariciones de este género en la ciudad fueron esporádicas hasta 2005, a partir de ese año se observó una frecuencia de denuncias constantes, lo que podría sugerir en ese periodo el establecimiento de las poblaciones. Aunque OjangurenAffilastro et al. (2019) reportaron formalmente la presencia de T. confluens en la ciudad de La Plata en el 2018, esta especie ya había sido documentada en las consultas registradas en el Laboratorio de Aracnología desde mayo de 2005 y confirmada en este estudio.
El análisis de la información del CEPAVE indicaría que las dos poblaciones aquí estudiadas se habrían asentado en dos momentos diferentes. En la Zona II de la ciudad, las denuncias fueron sostenidas y en aumento desde 2005, mientras en la zona Zona I las denuncias comenzaron en 2011 y desde ese momento y hasta la actualidad se ha observado una presencia creciente, lo que podría asociarse a la gran remoción del terreno por la construcción de viviendas en esta zona y por consecuencia la destrucción de los lugares donde habitan los escorpiones. Por otro lado, este crecimiento es coincidente con lo descripto para otras poblaciones del género Tityus en Argentina (Blanco et al. 2016; de Roodt et al. 2019). Blanco et al. (2016) señalan que la distribución en Ciudad Autónoma de Buenos Aires se daría por las redes de subterráneos y que la presencia de T. carrilloi se asocia a las estaciones ferroviarias. La ciudad de La Plata no cuenta con líneas subterráneas de trenes, pero la distribución podría estar asociada a los desagües pluviales de esta ciudad. El análisis de la dispersión independiente en las dos zonas podría indicar que este sería el camino debido a que estas redes son inconexas entre sí. Nuestros datos también coinciden con los de Blanco et al. (2016) en encontrar a T. carrilloi asociado a estaciones ferroviarias, y con lo de Maury (1997) donde sostiene que este género utilizaría a los durmientes como medio de dispersión. La estación Gambier dejó de estar activa como estación de pasajeros en el año 1977 y sus talleres pasaron a ser depósito de durmientes provenientes del desarme de vías de diferentes lugares, siendo utilizados como tal hasta el día de hoy. Dado que el asentamiento de la población del género Tityus se habría producido desde 2011 en adelante para la Zona I, es posible que estos individuos hayan sido transportados en los durmientes de madera depositados en los talleres y así colonizar las viviendas a medida que avanzó y avanza la construcción de nuevos barrios en esa zona.

Diferentes autores propusieron que algunas especies de este género tienen reproducción partenogenética, confirmada para T. carrilloi y sugerida para T. confluens (Francke 2008, Maury 1997, Ojanguren-Affilastro et al. 2021, Toscano-Gadea 2004). Esta hipótesis se vería reforzada con la ausencia de machos en los ejemplares hallados en la ciudad en estos 15 años. Este tipo de reproducción favorecería el crecimiento de las poblaciones en ambientes urbanos con alto grado de estrés ambiental.

Nuestros resultados demuestran que el avance territorial del género Tityus en la ciudad de La Plata es creciente y sostenido, y está puesto de manifiesto en los valores de crecimiento tanto anual como históricos. Dada la importancia sanitaria del género y por tener hábitos sinantrópicos, se hace necesario concientizar a la población acerca de la presencia de estos arácnidos poniendo a su alcance estrategias de prevención y modo de acción ante posibles accidentes por picadura. Esto podría ser tanto para la comunidad de la ciudad de La Plata o cualquier otro centro urbano donde se ha asentado el género Tityus.

Es muy importante conocer los factores ambientales y antrópicos que influyen en la dispersión del géne- 
ro Tityus, ya que esto nos ayudará a predecir las nuevas zonas que podrían ser colonizadas. Esto, sumado al estudio del área de dispersión en un tiempo determinado, genera la posibilidad de adelantarse a los acontecimientos. Es habitual observar la problemática que se genera en una determinada zona, cuando los vecinos empiezan a encontrar ejemplares en sus hogares. Es fundamental entonces, poder prever esta circunstancia, y promover de manera adecuada la educación mediante talleres, charlas, etc., que fomenten la participación comunitaria. De igual manera, y debido a la potencialidad del veneno de este género de escorpiones, se hace necesaria la formación y capacitación del personal de salud, ya que sus picaduras podrían tornarse tema de consulta frecuente, con características regionales.

\section{Literatura citada}

Bibbs CS, Bengston SE, Gouge DH. 2014. Activity trends and movement distances in the Arizona bark scorpion (Scorpiones: Buthidae). Environmental Entomology 43(6):1613-1620. https://doi.org/10.1603/EN14148

Blanco G, Laskowicz R, Lanari L, Scarlato E, Damin C, Tito EH de, Roodt AR de. 2016. Distribución de los hallazgos de escorpiones en la Ciudad de Buenos Aires en el período 2001-2012 y sus implicancias sanitarias. Archivos Argentinos Pediatría 114(1):77-83. https://doi. org/10.5546/aap.2016.77

Diputados C De. 2014. Período 142o La Plata, 13 de agosto de 2014 9a. Reunión DIPUTADOS PRESENTES: 9a. SESION ORDINARIA PROVINCIA DE BUENOS AIRES [Internet]. [accessed 2021 May 21]. https://intranet. hcdiputados-ba.gov.ar/diarios/diario1429.pdf

Francke OF. 2008. A critical review of reports of parthenogenesis in Scorpions (Arachnida). Revista Ibérica Aracnología 16(c):93-104.

Maury EA. 1970. Redescripción y distribución en la argentina de Tityus trivittatus Kraepelin 1898 (Scorpiones, Buthidae) comentarios sobre sus hábitos domiciliarios y su peligrosidad. PHYSIS 29(79):405-421.

Maury EA. 1997. Tityus trivittatus en la Argentina Nuevos datos sobre distribución, partenogénesis, sinantropía y peligrosidad (Scorpiones, Buthidae). Publicaciones de extensión cultural y didáctica del Museo Argentino de Ciencias Naturales "Bernardino Rivadavia" 24:1-24.

Moyano RD. 2008. Aracnoidismo. Arañas y escorpiones de importancia Médica en Argentina. 1ra ed. Literature of Latin América LOLA, editor. Buenos Aires.

Ojanguren-Affilastro AA, Kochalka J, Guerrero-Orallana D, Garcete-Barrett B, de Roodt AR, Borges A, Ceccarelli FS. 2021. Redefinition of the identity and phylogenetic position of Tityus trivittatus Kraepelin 1898, and description of Tityus carrilloi n. sp. (Scorpiones; Buthidae), the most medically important scorpion of southern South America. Revista del Museo Argentino Ciencias Naturales, nueva serie 23(1):27-55. https:// doi.org/10.22179/REVMACN.23.714

Ojanguren-Affilastro A, Bizzoto C, Lanari L, Lenicov MR, Roodt A de. 2019. Presencia de Tityus confluens Borelli en la ciudad de Buenos Aires y expansión de la distribución de las especies de importancia médica de Tityus (Scorpiones; Buthidae) en la Argentina. Revista del Museo Argentino de Ciencias Naturales nueva serie. 21(1):101-112. https://doi.org/10.22179/REVMACN.21.638
Ojanguren-Affilastro, A.A. 2005. Estudio monográfico de los escorpiones de la República Argentina. Revista Ibérica de Aracnología 11: 75-246

Roewer CF. 1943. Úber eine neuerworbene Sammlung von Skorpionen des Natur-Museums Senckenberg. Senckenbergiana. 26(4):205-244.

de Roodt AR. 2014. Comments on environmental and sanitary aspects of the scorpionism by Tityus trivittatus in Buenos Aires City, Argentina. Toxins (Basel) 6(4):14341452. https://doi.org/10.3390/toxins6041434

de Roodt AR, García SI, Salomón OD, Segre L, Dolab JA, Funes RF, De Titto EH. 2003. Epidemiological and clinical aspects of scorpionism by Tityus trivittatus in Argentina. Toxicon 41(8):971-977. https://doi.org/10.1016/ S0041-0101(03)00066-7

de Roodt AR, Lanari LC, Remes-Lenicov M, Cargnel E, Damin CF, Greco V, Orduna TA, Lloveras S, Desio MA, van Grootheest JH, et al. 2019. Expansión de la distribución de escorpiones del género Tityus C. L. Koch 1836. Acta Toxicológica Argentina 27(3):109-119.

Toscano-Gadea CA. 2004. Confirmation of parthenogenesis in Tityus trivittatus Kraepelin 1898 (Scorpiones, Buthidae). The Journal of Arachnology 32:866-869. https:// doi.org/10.1636/S03-21.1

Agradecimientos / Acknowledgments:

Conflicto de intereses / Competing interests:

Los autores no incurren en conflictos de intereses.

\section{Rol de los autores / Authors Roles:}

LAG: realizó la conceptualización, metodología, validación, análisis formal, investigación, curación de los datos, escritura del manuscrito. SEG: realizó la conceptualización, metodología, validación, análisis formal, investigación, escritura del manuscrito. SGRG: realizó la conceptualización, metodología, validación, análisis formal, investigación, escritura del manuscrito. AG: que realizó la conceptualización, metodología, validación, análisis formal, investigación, escritura del manuscrito.

\section{Fuentes de financiamiento / Funding:}

Los autores declaran que esta investigación no recibió ninguna subvención específica de ninguna agencia de financiación.

\section{Aspectos éticos / legales; Ethics / legals:}

Los autores declaran que no violaron ni omitieron normas éticas o legales en esta investigación. Este trabajo no involucró colecta de ejemplares, sino que los mismos fueron el producto de las consultas que llegaban al Instituto CEPAVE. Por ser ejemplares de especies urbanas y de importancia sanitaria, las consultas con ejemplares vivos no pudieron ser devueltas al lugar de captura y los mismos se mantuvieron en cautiverio hasta su muerte. 